\title{
Comprehensive analysis of IncRNA and miRNA expression profiles and ceRNA network construction in negative pressure wound therapy
}

\author{
Jie $\mathrm{Wu}^{1 \#}$, Yong Qin ${ }^{2 \#}$, Zhirui $\mathrm{Li}^{3,4 \#}$, Jiantao $\mathrm{Li}^{4,5}$, Litao $\mathrm{Li}^{1,4}$, Sheng Tao ${ }^{1,4}$, Daohong Liu ${ }^{1,4,5}$ \\ ${ }^{1}$ Department of Orthopedics, The Eighth Medical Center of PLA General Hospital, Beijing, China; ${ }^{2}$ Department of Orthopedics, Second Affiliated \\ Hospital of Harbin Medical University, Heilongiiang, China; ${ }^{3}$ Department of Orthopedics, Hainan Hospital of PLA General Hospital, Sanya, China; \\ ${ }^{4}$ Senior Department of Orthopedics, The Fourth Medical Center of PLA General Hospital, Beijing, China; ${ }^{5}$ National Clinical Research Center for \\ Orthopedics, Sports Medicine \& Rehabilitation, Beijing, China \\ Contributions: (I) Conception and design: D Liu, S Tao; (II) Administrative support: None; (III) Provision of study materials or patients: D Liu, J Wu; \\ (IV) Collection and assembly of data: Z Li, J Li, L Li; (V) Data analysis and interpretation: J Wu, Y Qin; (VI) Manuscript writing: All authors; (VII) \\ Final approval of manuscript: All authors. \\ "These authors contributed equally to this work. \\ Correspondence to: Sheng Tao; Daohong Liu. Department of Orthopedics, The Eighth Medical Center of PLA General Hospital, Beijing, China. \\ Email: taoshphy@126.com; domb@vip.sina.com.
}

Background: This study aims to explore the molecular mechanism of negative pressure wound therapy (NPWT) at the transcriptome level through whole transcriptome sequencing and biometric analysis.

Methods: A rat skin defect model was constructed and randomly divided into a NPWT group and a gauze group. The tissue in the center of the wound was used for whole transcriptome sequencing, and differentially expressed messenger RNAs (DEmRNAs), long noncoding RNAs (DElncRNAs), and microRNAs (DEmiRNAs) were identified between the two groups. Quantitative real time-polymerase chain reaction (qRT-PCR) analysis was used to verify the sequencing results. Functional enrichment analysis, pathway analysis, and protein-protein interaction (PPI) network analysis of DEmRNAs were conducted. Through bioinformatics analysis, a lncRNA-associated competing endogenous RNA (ceRNA) network was identified and constructed.

Results: We detected 896 DEmRNAs, 1,471 DElncRNAs, and 20 DEmiRNAs between the two groups. qRT-PCR verified the sequencing results. Functional analysis showed that DEmRNAs were mainly enriched in immune system processes and the Notch signaling pathway. Protein tyrosine phosphatase receptor type C (PTPRC) and signal transducer and activator of transcription 1 (STAT1) were the central hub nodes in the PPI analysis. The ceRNA network contained 11 mRNAs, 15 lncRNAs, and 4 miRNAs.

Conclusions: We identified several DEmRNAs, DElncRNAs, and DEmiRNAs between the NPWT treatment group and the control group. These findings may provide new insights into the pathophysiological mechanism of NPWT and wound healing.

Keywords: Negative pressure wound therapy (NPWT); next-generation sequencing; transcriptome; noncoding RNAs

Submitted Jun 18, 2021. Accepted for publication Aug 16, 2021.

doi: 10.21037/atm-21-3626

View this article at: https://dx.doi.org/10.21037/atm-21-3626

\footnotetext{
^ ORCID: 0000-0002-6572-9762.
} 


\section{Introduction}

Negative pressure wound therapy (NPWT) is a revolutionary and innovative wound treatment for various complex wounds. Since its development in the 1990s (1), the therapy is now widely used in clinical treatment with favorable clinical results (2-5). NPWT promotes wound healing through multiple mechanisms. From a macro point of view, the benefits of NPWT include reducing edema by draining excess fluid from the wound, improving local blood flow, and reducing the wound area through locally formed retractive force (1). Further research has recognized that the physical effects of NPWT cause changes, such as the local inflammatory response state, oxygen concentration, and micromechanical environment, in the microenvironment of the wound (6). These series of changes affect the synthesis and secretion of growth factors, inflammatory cytokines and chemokines, ultimately promoting blood vessel formation and granulation tissue hyperplasia (7-9). These pathophysiological changes involve many genes. However, the molecular mechanism of NPWT has not yet been fully revealed.

Analyzing the mechanism of NPWT at the genetic level will provide not only a theoretical basis for the clinical use of NPWT but also a new way to identify novel targets and mechanisms for wound healing. Through whole genome microarray surveys, Derrick et al. discovered that, compared with those in moist wound healing and gauze under suction therapy, more genes were upregulated and there were increased healing-related pathways in wound tissue treated with NPWT (10).

In the human genome, a large number of RNAs, known as noncoding RNAs (ncRNAs), are not translated into proteins (11). Studies have shown that ncRNAs can account for more than $90 \%$ of the mammalian genome, and they play an important role in the process of proliferation, differentiation, and apoptosis $(12,13)$. Among them, microRNAs (miRNAs) are a class of ncRNAs 18-24 nucleotides in length that can bind to target messenger RNA (mRNA) molecules, known as miRNA response elements (MREs). These MREs reduce the expression of specific proteins at the posttranscriptional level by either preventing their translation or promoting their degradation (14). Long noncoding RNAs (lncRNAs) are ncRNAs that are more than 200 nucleotides in length. According to the competing endogenous RNA (ceRNA) hypothesis (15), lncRNAs can interact with mRNA by competitively binding their common miRNA and then regulating downstream gene expression at the translation level. The lncRNA-associated ceRNA network has been studied in many fields, including oncology $(16,17)$ and orthopedics $(18,19)$. However, there is currently no ceRNA research focus in NPWT.

To better understand the mechanism of NPWT at the transcriptome level, we established a rat skin defect model and randomly divided the animals into an experimental group and a control group, which were given negative pressure treatment and conventional gauze therapy, respectively. Whole transcriptome sequencing was used to systematically identify differentially expressed mRNAs, lncRNAs, and miRNAs between the two groups. We first conducted functional and pathway analyses of differentially expressed mRNAs and identified and constructed a lncRNA-associated ceRNA network in NPWT. We present the following article in accordance with the ARRIVE reporting checklist (available at https://dx.doi.org/10.21037/ atm-21-3626).

\section{Methods}

\section{Animals}

Experiments were performed under a project license (No. 309201906230928) granted by the Medical Ethics Committee of the Eighth Medical Center of PLA General Hospital, in compliance with national guidelines for the care and use of animals. A total of 6 male Sprague-Dawley (SD) rats (age: 8 weeks; weight: $260-300 \mathrm{~g}$ ) were obtained from the Experimental Animal Center of the 8th Medical Center of Chinese PLA General Hospital. The rats were randomly assigned to the NPWT group and gauze group, with 3 rats in each group. All rats were individually housed in special cages under conventional conditions with free access to food and water.

\section{Wound creation and animal grouping}

One day before the operation, a razor was used to shave the entire back of the experimental rats. An appropriate amount of depilatory agent was then evenly sprayed on the shaved area to obtain smooth and hairless skin. After hair removal, the rats was adaptively fed for 1 day. Rats were anesthetized by intraperitoneal injection of $40 \mathrm{mg} / \mathrm{kg}$ pentobarbital sodium (1\%) 10 minutes prior to the operation. The backs of all the rats were disinfected with povidone iodine solution and $75 \%$ medical alcohol. A $3.0 \mathrm{~cm}$ diameter full-thickness circular skin tissue was then excised down to the deep fascia 
to obtain skin defect models. All animals were randomly divided into the NPWT group (experimental group) or sterile gauze dressing group (control group). In the NPWT group, wounds were covered with a poly (vinyl alcohol) shrink formaldehyde bubble dressing and sealed with a biological semipermeable membrane (VSD Medical Science and Technology Co., Ltd., Wuhan, China). Continuous suction at a negative pressure of $125 \mathrm{mmHg}$ was provided by a portable negative pressure device. Negative pressure tightness was checked twice every day, and the NPWT dressings were changed every 48 hours, as per the manufacturer's recommendations. In the gauze group, wounds were covered with sterile gauze, and dressings were checked and changed daily.

The rats were killed by cervical dislocation on the fifth day after the operation. After removing the exudate on the wound surface with sterile saline solution, a scalpel was used to perform a biopsy in the center of the wound under aseptic conditions. Samples were then harvested and stored at $-80^{\circ} \mathrm{C}$ until time of analysis.

\section{RNA extraction}

According to the manufacturer's instructions, TRIzol agent (Invitrogen, USA) was used to extract total RNA. A trace nucleic acid protein analyzer (Analytik Jena, Jena, Germany) was used to detect the optical density (OD) value $260 \mathrm{~nm} / \mathrm{OD}$ $280 \mathrm{~nm}$ to identify the RNA sample concentration and eliminate the possibility of RNA contamination. A Bioanalyzer 2100 (Agilent, USA) was used to assess total RNA quality. RNA integrity number (RIN) $\geq 7$ and 260/280 $\geq 1.8$ were used as thresholds. RNase-free DNase I (Ambion Inc., USA) was used to eliminate potential genomic DNA contamination. RNAs were preserved at $-80^{\circ} \mathrm{C}$.

\section{Library preparation, examination, and clustering and sequencing}

RNA sequencing was performed by CapitalBio Technology (Beijing, China). The researchers who sequenced the samples were unaware of the treatment group assignment. A total RNA amount of $3 \mu \mathrm{g}$ per sample was used as initial material for the RNA sample preparations. Epicentre RiboZero Magnetic Kits (Epicentre, USA) were used to remove ribosomal RNA. According to the manufacturer's procedure, the Next Ultra RNA Library Prep Kit for Illumina (New England Biolabs, USA) was used to generate the sequencing libraries. A Qubit DNA HS Assay Kit (Invitrogen, USA) was used to accurately quantify polymerase chain reaction (PCR) products. A cBot cluster generation system (Illumina, USA) was used to cluster the index-coded samples. The libraries were subsequently sequenced on an Illumina HiSeq 4000 platform (Illumina, USA).

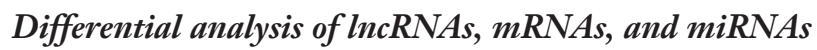

FastQC (v0.11.2) software was used to filter the original sequencing results to obtain high-quality data suitable for analysis. Limma (v. 3.32.10) software was used for differential gene expression analysis. Differentially expressed mRNAs (DEmRNAs), differentially expressed lncRNAs (DElncRNAs), and differentially expressed miRNAs (DEmiRNAs) were determined according to the following criteria: $\mathrm{P} \leq 0.05$ and $\mid \log$ fold change (FC) $\mid \geq 1$. Ggplot2 software was used to make the volcano maps and heatmaps.

\section{Quantitative real-time PCR analysis}

Quantitative real-time PCR (qRT-PCR) was used to validate the DElncRNAs. Four DElncRNAs were randomly selected. A Prime-Script RT reagent kit (TaKaRa, Japan) was used to reverse transcribe RNA to complement DNA (cDNA). The supplementary table (available online: https:// cdn.amegroups.cn/static/public/atm-21-3626-1.xlsx) shows the primers. GAPDH was chosen as an internal control for the lncRNAs. The experiment was repeated 3 times for each sample. The results were calculated by the $2^{-\Delta \Delta C t}$ method to compare the expression of lncRNAs between the two groups.

\section{Function and patbway analysis of DEmRNAs}

Gene Ontology (GO) is the functional significance analysis of genes and includes three major categories: biological processes (BP), cellular components (CC), and molecular functions (MF). Our research used BP within GO analysis to obtain the main functions of the differentially expressed genes. Kyoto Encyclopedia of Genes and Genomes (KEGG) pathway analysis was used to identify the significant pathways for differentially expressed genes.

The DAVID (the database for annotation, visualization and integrated discovery) Database (v. 6.8) was used to conduct GO analysis and KEGG pathway analysis. Hypergeometric distribution was used to perform hypothesis testing to obtain the $\mathrm{P}$ value of the enrichment 

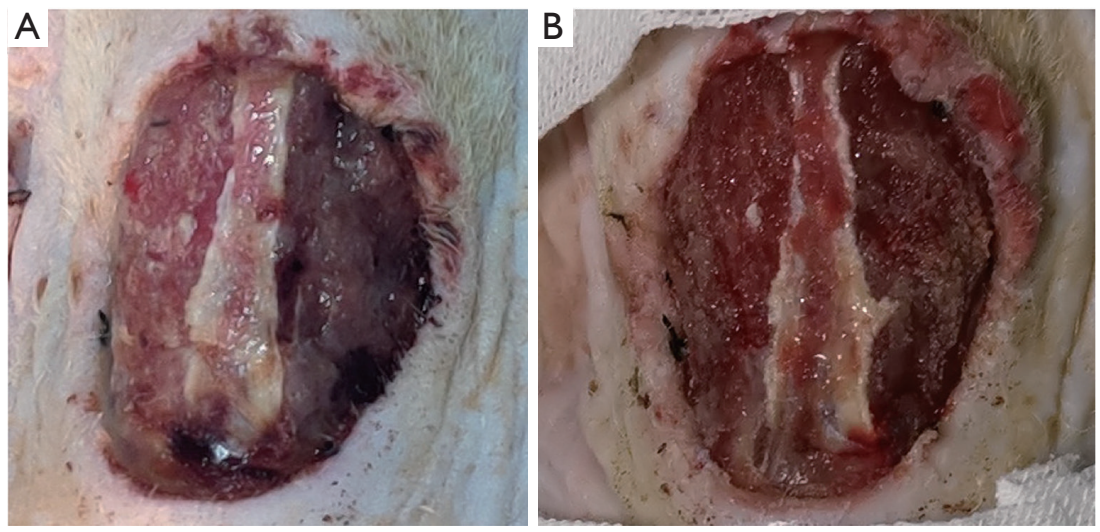

Figure 1 General observation of wounds of in the gauze group (A) and NPWT group (B). NPWT, negative pressure wound therapy.

result. To reduce the false-positive rate, multiple hypothesis testing was used to correct the $\mathrm{P}$ value to the obtain $\mathrm{q}$ value (the corrected $\mathrm{P}$ value). A q value $\leq 0.05$ indicated significant enrichment.

\section{Protein-protein interaction (PPI) network analysis of DEmRNAs}

Proteins are the biological basis of life and are responsible for various functions within the body. STRING (search tool for the retrieval of interacting genes/proteins) is a protein interaction database that can be used to analyze known and predicted protein interactions. The STRING database was used for PPI analysis, and the results were filtered based on the combined score. PPI pairs with a combined score of $>0.93$ were identified. The PPI network was then constructed using Cytoscape (v. 3.8.2). The top 20 relationship pairs with the highest network topology property indicators were considered to be hub nodes and were further analyzed.

\section{Construction of a lncRNA-associated ceRNA network}

The differentially expressed lncRNAs, miRNAs, and mRNAs obtained from RNA-sequencing data of the two groups were analyzed. The Pearson correlation coefficient (PCC) of differential lncRNAs and mRNAs was calculated, and significantly related lncRNA-mRNA interaction pairs were identified. The default filtering threshold was 0.99 . The Holm algorithm was used for the hypothesis test of the $\mathrm{P}$ value correction with a default filtering threshold of 0.05 . The miRanda tools were used to identify miRNA-mRNA and miRNA-lncRNA interaction pairs. Based on the analysis results, a ceRNA network of IncRNA-miRNA-mRNA was constructed and then visualized via Cytoscape v. 3.7.0 software.

\section{Statistical analysis}

Data were analyzed by using SPSS software (v. 19.0, IBM Corporation). The differences were compared through independent samples $t$-tests. A P value of $\leq 0.05$ was regarded as statistically significant.

\section{Results}

\section{General observations}

The rat wound model was successfully established, with two groups being treated (Figure S1). All 6 rats survived until the completion of the experiment, and there were no obvious signs of wound infection. Through general observation, it was found that both groups of wounds had granulation tissue formation, which was more apparent in the NPWT group (Figure 1).

\section{Differentially expressed $m R N A s, \operatorname{lncRNAs}$, and miRNAs}

We identified 896 DEmRNAs between the NPWT group and gauze group, of which 473 were upregulated and 423 were downregulated (available online: https://cdn. amegroups.cn/static/public/atm-21-3626-1.xlsx). A total of 1471 DElncRNAs were found (715 upregulated and 756 downregulated) (available online: https://cdn.amegroups. 


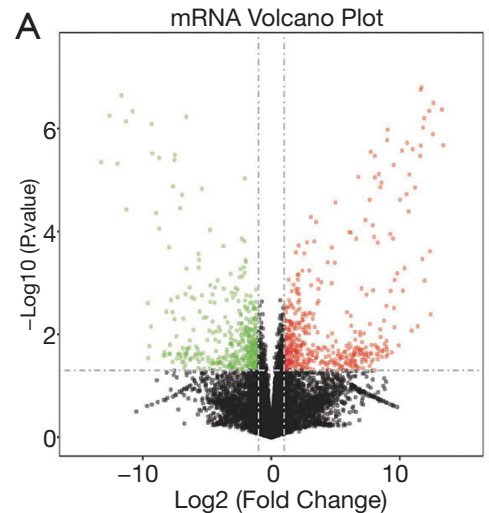

$\mathrm{D}$

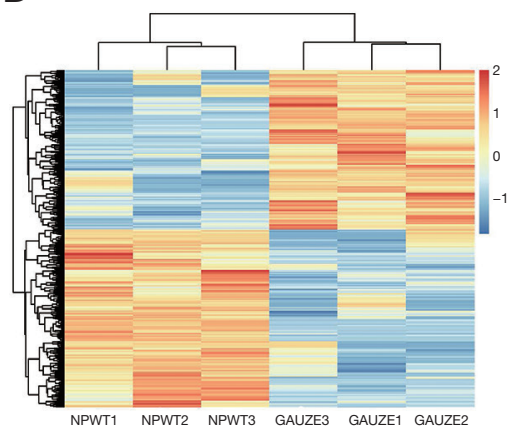

B

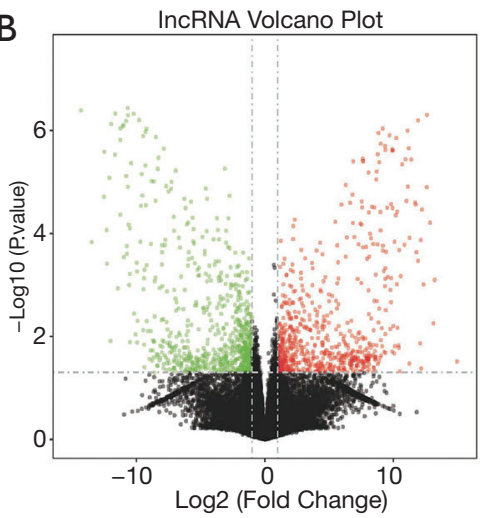

$E$

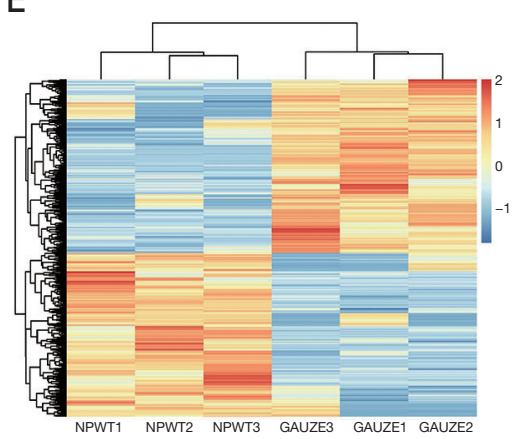

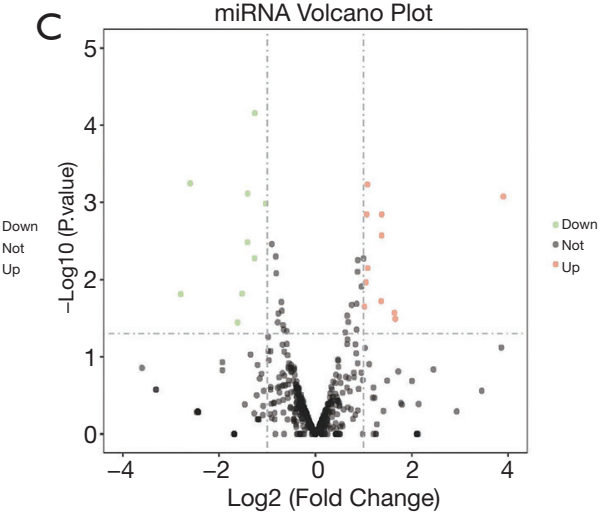

$\mathrm{F}$

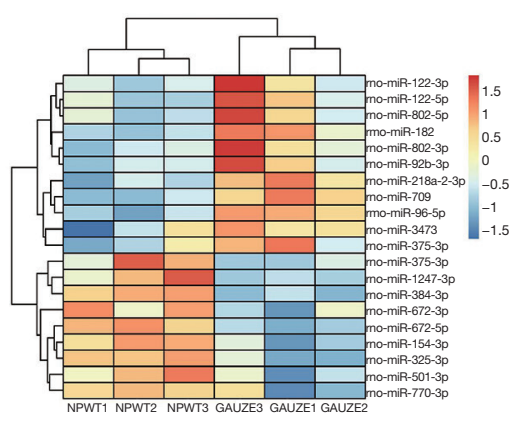

Figure 2 The expression profiles of differentially expressed mRNAs, lncRNAs, and miRNAs between the NPWT group and gauze group. Volcano plots of DEmRNAs (A), DElncRNAs (B), and DEmiRNAs (C) between the NPWT group and gauze group. The heatmap represents hierarchical clustering for DEmRNAs (D), DEIncRNAs (E), and DEmiRNAs (F) between the NPWT group and the gauze group. DEmRNA, differentially expressed messenger RNA; DElncRNA, differentially expressed long noncoding RNA; DEmiRNA, differentially expressed microRNA; NPWT, negative pressure wound therapy.

cn/static/public/atm-21-3626-2.xlsx). miRNA analysis also identified 20 DEmiRNAs, including 11 upregulated and 9 downregulated DEmiRNAs (Table S1). Volcano maps and heatmaps (Figure 2) were used to display the results.

\section{Quantitative RT-PCR analysis}

To verify the results of transcriptome sequencing screening, we conducted RT-PCR experiments to confirm the differential analysis at the molecular level, and 4 DElncRNAs in the top 10 significantly DElncRNAs were randomly selected. The primer sequence is shown in Table S2. The results showed that in the NPWT group, MERGE.27685.3 and NONRATT007883.2 were upregulated. Meanwhile, MERGE.6154.9 and MERGE.18820.19 were downregulated in the NPWT group (Figure 3). The RT-PCR results were consistent with the RNA-sequencing results.

\section{Functional enrichment analysis of differentially expressed $m R N A s$}

DEmRNAs underwent GO analysis and KEGG pathway analysis. The top 10 significantly enriched biological processes were determined using GO analysis with results shown in Figure 4A. The data indicated that DEmRNAs were mainly enriched in RNA processing, virus response, the Notch signaling pathway, negative regulation of $\mathrm{T}$ cell proliferation, and immune response. The top 10 significantly enriched KEGG pathway terms are shown in Figure 4B. Pathways were identified as markedly enriched in viral myocarditis, systemic lupus erythematosus, 

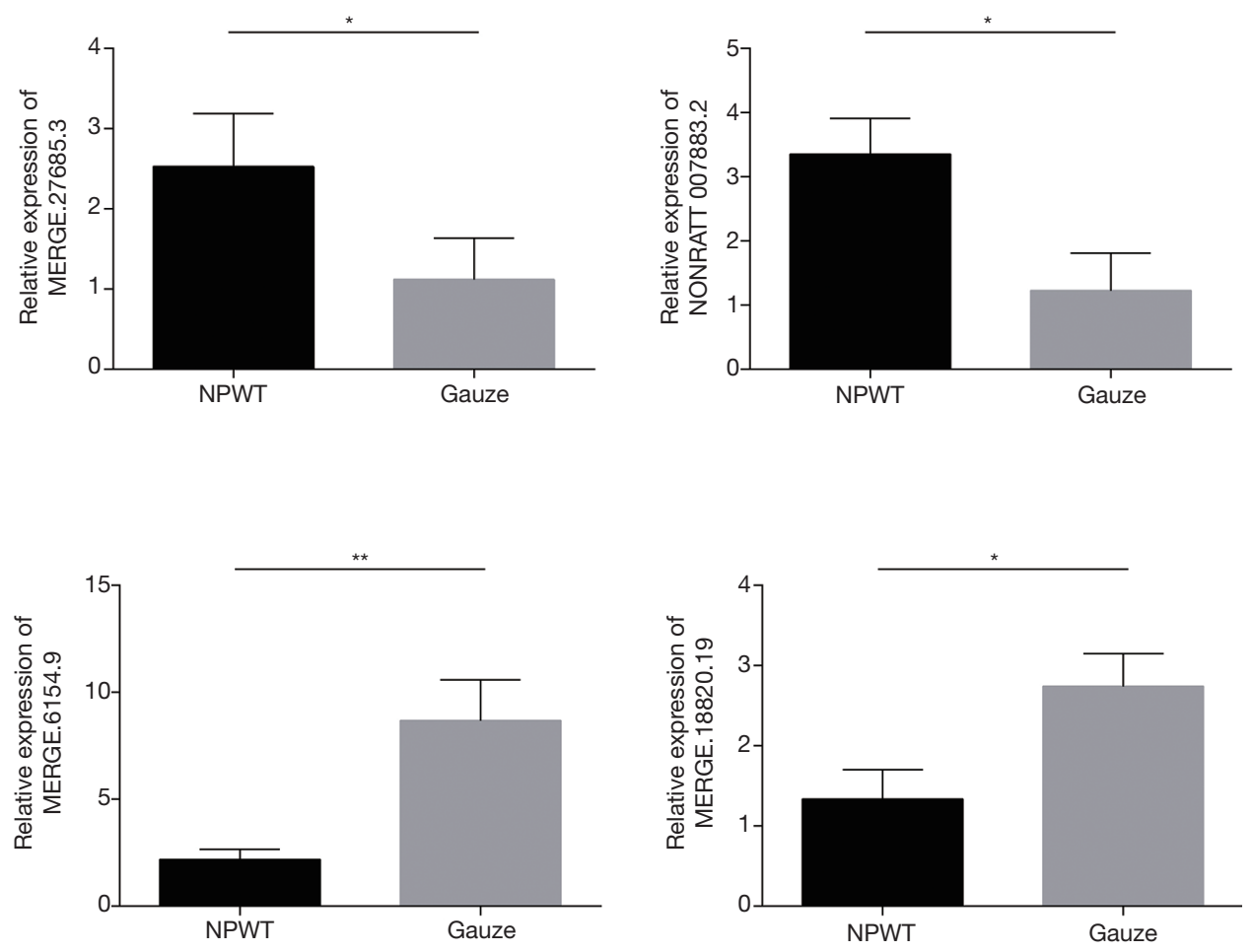

Figure 3 Validation of sequencing results by qRT-PCR. GAPDH was chosen as a housekeeping internal control. The $2^{-\Delta \Delta C t}$ method was used to conduct relative-quantitative calculations. The data are presented as the mean $\pm \operatorname{SEM}(\mathrm{n}=3) ;{ }^{*} \mathrm{P}<0.05,{ }^{* *} \mathrm{P}<0.01$.
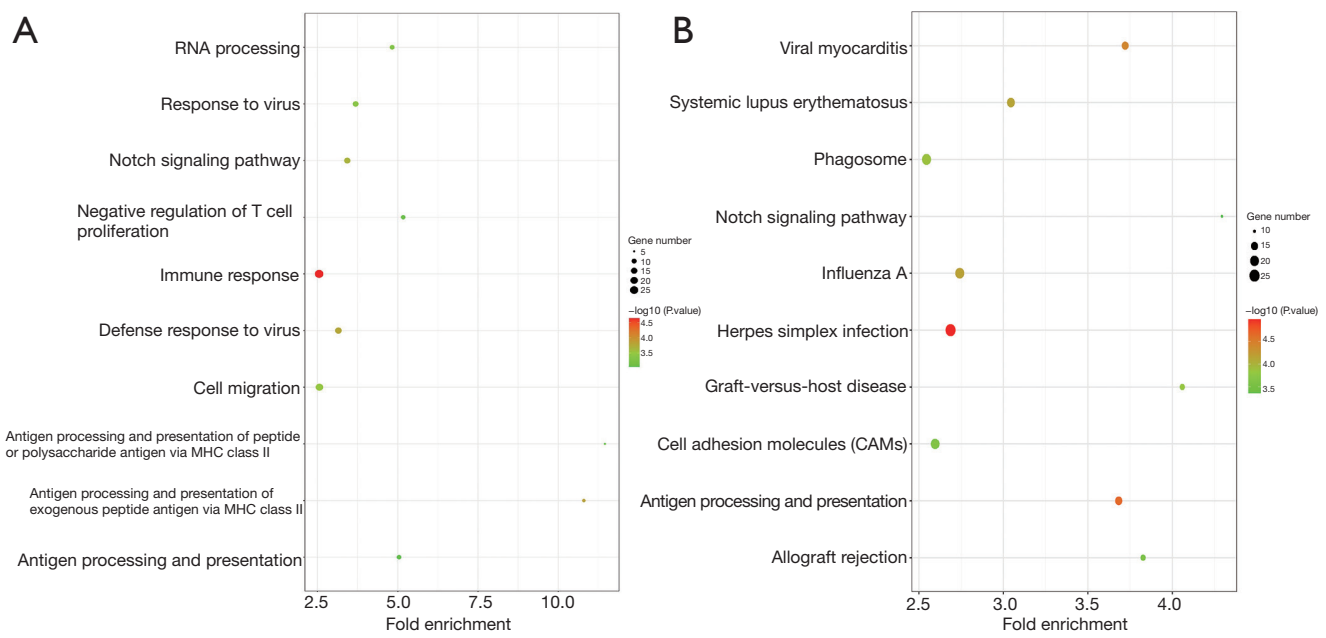

Figure 4 The results of functional and pathway analysis of DEmRNAs. (A) Shows the top 10 significantly enriched biological processes. (B) Shows the top 10 significantly enriched pathways. DEmRNA, differentially expressed messenger RNA.

phagosome, the Notch signaling pathway, and influenza A. Construction of the PPI network

The top 100 nodes ranked by degree and node relation within these nodes were chosen to establish the PPI network (Figure 5). According to the ranking of network topology property indicators of degree centrality, betweenness centrality, and closeness centrality, the top 20 nodes were separately identified as hub nodes (Table 1, 


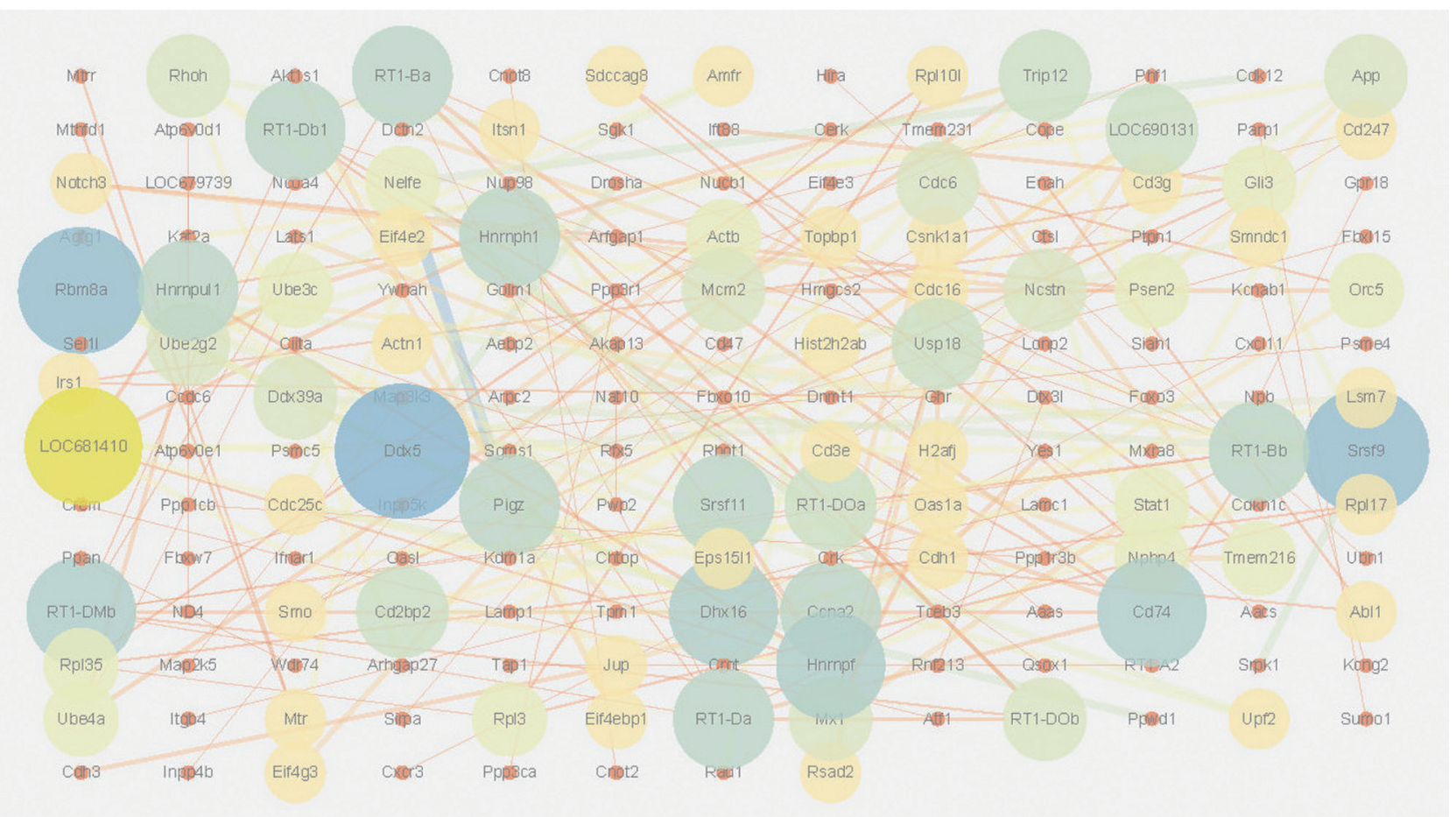

Figure 5 PPI network of DEmRNAs between the NPWT group and gauze group. PPI, protein-protein interaction; DEmRNA, differentially expressed messenger RNA; NPWT, negative pressure wound therapy.

Figure 6). Among these hub nodes, Trip12, PTPRC, and STAT1 were the central hub nodes that had the most neighboring connections to other coding genes.

\section{Construction of the lncRNA-miRNA-mRNA ceRNA regulatory network}

After separately identifying lncRNA-mRNA, miRNAmRNA, and miRNA-lncRNA interaction pairs, the pairs were integrated to construct the lncRNA-miRNA-mRNA regulatory network. The ceRNA network contained 15 lncRNAs, 11 mRNAs, and 4 miRNAs (Figure 7).

\section{Discussion}

Studying the mechanisms of NPWT at the genomic level will help uncover the molecular processes involved in wound healing, thereby improving NPWT. Researchers initially used gene microarrays to study NPWT at the gene level (10). Borys et al. (20) assessed the effect of NPWT on gene expression using human gene expression arrays. Genomics and transcriptomics have developed rapidly since the emergence of high-throughput sequencing technology.
This has allowed for the identification and annotation of many ncRNAs. Some of these ncRNAs have been shown to be involved in the regulation of inflammation, angiogenesis, and other biological processes related to wound healing. Zhang et al. (21) found that the downregulation of lncRNA Gas 5 can reduce protein expression of matrix metalloproteinase 7 (MMP-7), cleaved caspase- 3 and interleukin $1 \beta$ (IL-1 $\beta$ ), thereby reducing local inflammation. Furthermore, Du et al. (22) proposed that lncRNA Mirt2 is a negative regulator of inflammation. miRNAs play a role in inflammation by regulating cytokines such tumor necrosis factor-a (TNF-a), transforming growth factor- $\beta$ (TGF- $\beta$ ), and IL-10 (23). In addition, IncRNAs $(24,25)$ and miRNAs (23) regulate angiogenesis by targeting factors such as hypoxia-inducible factor (HIF) and vascular endothelial growth factor (VEGF).

In this study, we explored the role of NPWT in promoting wound healing at the transcriptome level. We used high-throughput sequencing to identify a large number of mRNAs and IncRNAs that differed between the two groups. The sequencing results were verified through RT-PCR experiments. Functional enrichment analysis was conducted to define the functions of DEmRNAs. The 
Table 1 Top 20 nodes of the PPI network ranked by network topology property indicators of degree centrality

\begin{tabular}{|c|c|}
\hline Name & Scores \\
\hline Thyroid Hormone Receptor Interactor 12 (TRIP12) & 42 \\
\hline Protein Tyrosine Phosphatase Receptor Type C (PTPRC) & ) 37 \\
\hline $\begin{array}{l}\text { Signal Transducer And Activator Of Transcription } 1 \\
\text { (STAT1) }\end{array}$ & 37 \\
\hline Amyloid Beta Precursor Protein (APP) & 35 \\
\hline DNA Methyltransferase 1 (DNMT1) & 34 \\
\hline $\begin{array}{l}\text { Phosphatidylinositol Glycan Anchor Biosynthesis Class } \\
\text { Z (PIGZ) }\end{array}$ & 33 \\
\hline Histone Cluster 1 H3 Family Member C (HIST1H3C) & 33 \\
\hline DEAD-Box Helicase 5 (DDX5) & 32 \\
\hline RNA Binding Motif Protein 8A (RBM8A) & 29 \\
\hline Cell Division Cycle 16 (CDC16) & 28 \\
\hline Ubiquitination Factor E4A (UBE4A) & 27 \\
\hline Cell Division Cycle 6 (CDC6) & 26 \\
\hline $\begin{array}{l}\text { Heterogeneous Nuclear Ribonucleoprotein H1 } \\
\text { (HNRNPH1) }\end{array}$ & 26 \\
\hline Deltex E3 Ubiquitin Ligase 3L (DTX3L) & 25 \\
\hline Insulin receptor substrate 1 (IRS1) & 25 \\
\hline MX Dynamin Like GTPase 1(MX1) & 25 \\
\hline Clathrin Heavy Chain (CLTC) & 25 \\
\hline Actin Beta (ACTB) & 24 \\
\hline CD3g Antigen, Gamma Polypeptide (CD3G) & 23 \\
\hline Ribosomal Protein L3 (RPL3) & 23 \\
\hline
\end{tabular}

PPI, protein-protein interaction.

results of GO analysis showed that DEmRNAs regulated immune system processes, which are important and complex biological processes in wound healing $(26,27)$. Negative regulation of $\mathrm{T}$ cell proliferation, another significantly enriched biological process, was involved in wound repair through immunological pathways. The Notch signaling pathway was significantly enriched in both GO analysis and KEGG analysis. The Notch signaling pathway is a highly conserved intercellular signal transduction pathway, which is widely involved in the differentiation, proliferation, and apoptosis processes of various cells (28). It is associated with $\mathrm{T}$ cell-mediated immune responses, inflammation, and hematopoiesis (29). Chigurupati et al. (30) found that Notch signaling affects multiple cell types involved in wound healing, influencing the enhancement of vascular endothelial cell proliferation, migration and tube formation, the regulation of local inflammatory responses, and the behaviors of keratinocytes and fibroblasts. Shi et al. (31) found that Notch signaling plays important roles in wound repair by modulating the target genes hairy and enhancer of split 1 (HES1). In addition, many studies have shown that the Notch signaling pathway is related to wound healing (32-34). Given the findings of previous studies, along with our own results, we can surmise that the Notch signaling pathway may be the key pathway by which NPWT regulates wound healing.

Proteins interact with each other in all organisms. These protein interaction networks form the basis of all biological processes and include biological signal transmission, gene regulation, energy and material metabolism, and cell cycle regulation. PPI networks were constructed for DEmRNAs, leading to the identification of hub genes. Protein tyrosine phosphatase receptor type C (PTPRC; CD45) is a key molecule of signal transduction in the cell membrane and plays a key role in the development, maturation, functional regulation, and signal transmission of immune cells $(35,36)$. There are no previous studies on the effect of PTPRC on wound healing. We propose that PTPRC may affect wound healing through immune regulation and inflammation.

Signal transducer and activator of transcription 1 (STAT1), an important signal transcription factor, can be activated by a range of cytokines and growth factors, including interferon-alpha, interferon-gamma, Epidermal growth factor (EGF), platelet derived growth factor (PDGF), and IL6 (37-39). STAT1 can promote apoptosis and inflammation (40). Moreover, Medley et al. found that STAT1 plays a role in wound repair as a fibroblastmediated inhibitor (41). Along with previous studies, our findings indicate that STAT1 may participate in wound repair through the inflammatory process and the regulation of fibroblasts.

To further analyze the mechanism of NPWT, we constructed a ceRNA network through biometric analysis: 11 mRNAs, 15 lncRNAs, and 4 miRNAs were included in the ceRNA. Among the genes which have been previously studied and reported, miRNA-218 has been found to be an important intron miRNA that regulates angiogenesis in tumors $(42,43)$. miRNA 3473 plays a role in inflammation through the PI3K/Akt signaling pathway (44), while miRNA-672-5p is involved in angiogenesis and adipocyte regulation through ANGPT14 target genes (45). lncRNAs can participate in the wound healing process by 


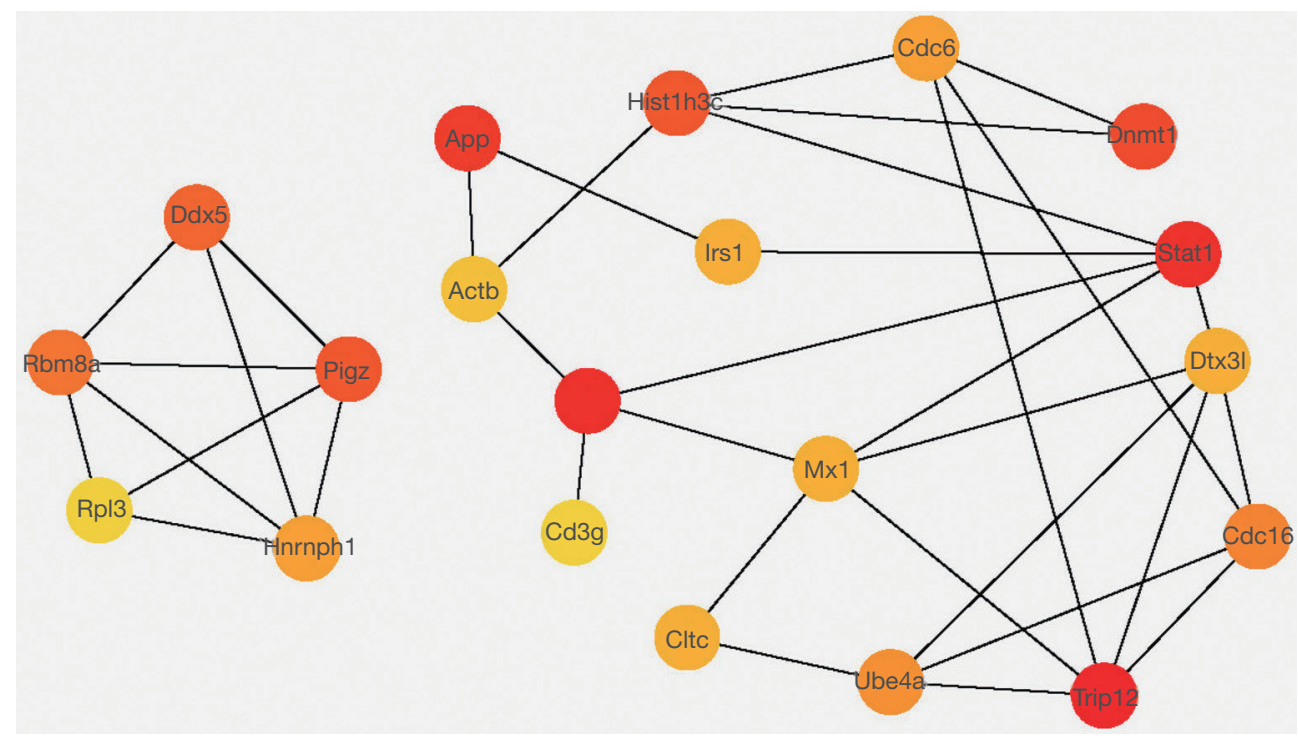

Figure 6 The top 20 nodes of the PPI network and their interrelationships. PPI, protein-protein interaction.

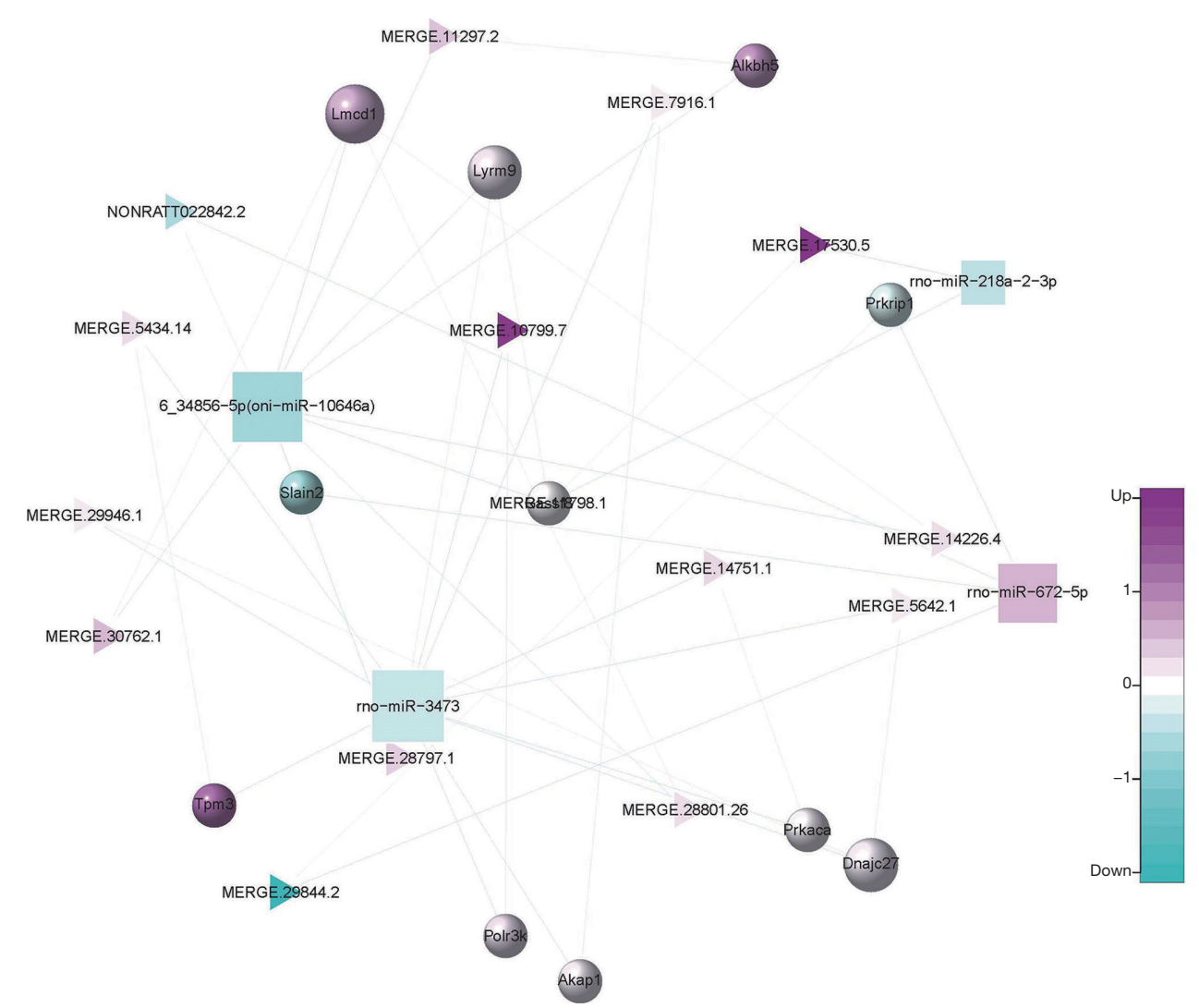

Figure 7 The mRNA-miRNA-lncRNA ceRNA regulatory network. Triangles represent lncRNAs, circular nodes represent mRNAs, and rectangular nodes represent miRNAs. mRNA, messenger RNA; miRNA, microRNA; lncRNA, long noncoding RNA; ceRNA, competing endogenous RNA. 
competitively binding corresponding miRNAs.

It should be noted that we have conducted only a preliminary exploration of the ceRNA network in NPWT. Other limitations of this study include the insufficient sample size and the use of rats as the experimental animals, which restricted the comprehensiveness of the relevant database and led to some lncRNAs being unannotated, impacting result analyses. Finally, this study was conducted on the fifth day of treatment. Because wound healing is a continuously developing process, subsequent studies on the entire progression of healing are still needed.

\section{Conclusions}

We identified a number of DEmRNAs, DElncRNAs, and DEmiRNAs between the NPWT group and the gauze group. The Notch pathway may be a critical signal transduction pathway through which NPWT affects wound healing. These findings may provide new insights into the pathophysiological mechanism of NPWT and wound healing.

\section{Acknowledgments}

Funding: This study was supported by the Major Project of Military Logistics Research (No. AWS17J004-02-13).

\section{Footnote}

Reporting Checklist: The authors have completed the ARRIVE reporting checklist. Available at https://dx.doi. org/10.21037/atm-21-3626

Data Sharing Statement: Available at https://dx.doi. org/10.21037/atm-21-3626

Conflicts of Interest: All authors have completed the ICMJE uniform disclosure form (available at https://dx.doi. org/10.21037/atm-21-3626). The authors have no conflicts of interest to declare.

Ethical Statement: The authors are accountable for all aspects of the work in ensuring that questions related to the accuracy or integrity of any part of the work are appropriately investigated and resolved. Experiments were performed under a project license (No. 309201906230928) granted by the Medical Ethics Committee of the Eighth Medical Center of PLA General Hospital, in compliance with national guidelines for the care and use of animals.

Open Access Statement: This is an Open Access article distributed in accordance with the Creative Commons Attribution-NonCommercial-NoDerivs 4.0 International License (CC BY-NC-ND 4.0), which permits the noncommercial replication and distribution of the article with the strict proviso that no changes or edits are made and the original work is properly cited (including links to both the formal publication through the relevant DOI and the license). See: https://creativecommons.org/licenses/by-nc-nd/4.0/.

\section{References}

1. Argenta LC, Morykwas MJ. Vacuum-assisted closure: a new method for wound control and treatment: clinical experience. Ann Plast Surg 1997;38:563-76; discussion 577.

2. Szmyt K, Łukasz K, Bobkiewicz A, et al. Comparison of the effectiveness of the treatment using standard methods and negative pressure wound therapy (NPWT) in patients treated with open abdomen technique. Pol Przegl Chir 2015;87:22-30.

3. Eyvaz K, Kement M, Balin S, et al. Clinical evaluation of negative-pressure wound therapy in the management of electrical burns. Ulus Travma Acil Cerrahi Derg 2018;24:456-61.

4. Blum ML, Esser M, Richardson M, et al. Negative pressure wound therapy reduces deep infection rate in open tibial fractures. J Orthop Trauma 2012;26:499-505.

5. Sorrentino VP, Corte AD, Fattopace A, et al. The use of negative pressure wound therapy (npwt) and dermal substitutes in the treatment of diabetic foot ulcers. BMC Geriatr 2011;11:A39.

6. Borys S, Hohendorff J, Frankfurter C, et al. Negative pressure wound therapy use in diabetic foot syndromefrom mechanisms of action to clinical practice. Eur J Clin Invest 2019;49:e13067.

7. Liu D, Li Z, Wang G, et al. Virulence analysis of Staphylococcus aureus in a rabbit model of infected fullthickness wound under negative pressure wound therapy. Antonie Van Leeuwenhoek 2018;111:161-70.

8. Ma Z, Li Z, Shou K, et al. Negative pressure wound therapy: Regulating blood flow perfusion and microvessel maturation through microvascular pericytes. Int J Mol Med 2017;40:1415-25.

9. Liu D, Zhang L, Li T, et al. Negative-pressure wound therapy enhances local inflammatory responses in acute infected soft-tissue wound. Cell Biochem Biophys 2014;70:539-47. 
10. Derrick KL, Norbury K, Kieswetter K, et al. Comparative analysis of global gene expression profiles between diabetic rat wounds treated with vacuum-assisted closure therapy, moist wound healing or gauze under suction. Int Wound J 2008;5:615-24.

11. Ambros V. The functions of animal microRNAs. Nature 2004;431:350-5.

12. Bartel DP. MicroRNAs: target recognition and regulatory functions. Cell 2009;136:215-33.

13. Ren D, Cai Y, Xu G. Potential of microRNA expression profile in predicting renal impairment risk in multiple myeloma patients. Transl Cancer Res 2020;9:1495-505.

14. Gennari L, Bianciardi S, Merlotti D. MicroRNAs in bone diseases. Osteoporos Int 2017;28:1191-213.

15. Salmena L, Poliseno L, Tay Y, et al. A ceRNA hypothesis: the Rosetta Stone of a hidden RNA language? Cell 2011;146:353-8.

16. Chen Y, Sun F, Zhang Y, et al. Comprehensive molecular characterization of circRNA-associated ceRNA network in constrictive pericarditis. Ann Transl Med 2020;8:549.

17. Li Y, Wang H, Huang H. Long non-coding RNA MIR205HG function as a ceRNA to accelerate tumor growth and progression via sponging miR-122-5p in cervical cancer. Biochem Biophys Res Commun 2019;514:78-85.

18. Chen H, Chen L. An integrated analysis of the competing endogenous RNA network and co-expression network revealed seven hub long non-coding RNAs in osteoarthritis. Bone Joint Res 2020;9:90-8.

19. Zhang X, Liang H, Kourkoumelis N, et al. Comprehensive Analysis of lncRNA and miRNA Expression Profiles and ceRNA Network Construction in Osteoporosis. Calcif Tissue Int 2020;106:343-54.

20. Borys S, Ludwig-Slomczynska AH, Seweryn M, et al. Negative pressure wound therapy in the treatment of diabetic foot ulcers may be mediated through differential gene expression. Acta Diabetol 2019;56:115-20.

21. Zhang Z, Li X, Chen F, et al. Downregulation of LncRNA Gas5 inhibits apoptosis and inflammation after spinal cord ischemia-reperfusion in rats. Brain Res Bull 2021;168:110-9.

22. Du M, Yuan L, Tan X, et al. The LPS-inducible lncRNA Mirt2 is a negative regulator of inflammation. Nat Commun 2017;8:2049.

23. Roy S, Sen CK. miRNA in wound inflammation and angiogenesis. Microcirculation 2012;19:224-32.

24. Zhang B, Wang D, Ji TF, et al. Overexpression of lncRNA ANRIL up-regulates VEGF expression and promotes angiogenesis of diabetes mellitus combined with cerebral infarction by activating $\mathrm{NF}-\kappa \mathrm{B}$ signaling pathway in a rat model. Oncotarget 2017;8:17347-59.

25. Kumar MM, Goyal R. LncRNA as a Therapeutic Target for Angiogenesis. Curr Top Med Chem 2017;17:1750-7.

26. Koh TJ, DiPietro LA. Inflammation and wound healing: the role of the macrophage. Expert Rev Mol Med 2011;13:e23.

27. Park JE, Barbul A. Understanding the role of immune regulation in wound healing. Am J Surg 2004;187:11S-6S.

28. Radtke F, Fasnacht N, Macdonald HR. Notch signaling in the immune system. Immunity 2010;32:14-27.

29. Shang Y, Smith S, Hu X. Role of Notch signaling in regulating innate immunity and inflammation in health and disease. Protein Cell 2016;7:159-74.

30. Chigurupati S, Arumugam TV, Son TG, et al. Involvement of notch signaling in wound healing. PLoS One 2007;2:e1167.

31. Shi Y, Shu B, Yang R, et al. Wnt and Notch signaling pathway involved in wound healing by targeting c-Myc and Hes1 separately. Stem Cell Res Ther 2015;6:120.

32. Takazawa Y, Ogawa E, Saito R, et al. Notch downregulation in regenerated epidermis contributes to enhanced expression of interleukin-36 $\alpha$ and suppression of keratinocyte differentiation during wound healing. J Dermatol Sci 2015;79:10-9.

33. Syed F, Bayat A. Notch signaling pathway in keloid disease: enhanced fibroblast activity in a Jagged-1 peptidedependent manner in lesional vs. extralesional fibroblasts. Wound Repair Regen 2012;20:688-706.

34. Lu H, Lu Q, Zheng Y, et al. Notch signaling promotes the corneal epithelium wound healing. Mol Vis 2012;18:403-11.

35. Hermiston ML, Xu Z, Weiss A. CD45: a critical regulator of signaling thresholds in immune cells. Annu Rev Immunol 2003;21:107-37.

36. Jacobsen M, Hoffmann S, Cepok S, et al. A novel mutation in PTPRC interferes with splicing and alters the structure of the human CD45 molecule. Immunogenetics 2002;54:158-63.

37. Klampfer L, Huang J, Swaby LA, et al. Requirement of histone deacetylase activity for signaling by STAT1. J Biol Chem 2004;279:30358-68.

38. Bromberg JF, Horvath CM, Wen Z, et al. Transcriptionally active Stat1 is required for the antiproliferative effects of both interferon alpha and interferon gamma. Proc Natl Acad Sci U S A 1996;93:7673-8.

39. Canaff L, Zhou X, Hendy GN. The proinflammatory cytokine, interleukin-6, up-regulates calcium-sensing 
receptor gene transcription via Stat $1 / 3$ and $\mathrm{Sp} 1 / 3$. J Biol Chem 2008;283:13586-600.

40. Moore F, Naamane N, Colli ML, et al. STAT1 is a master regulator of pancreatic $\{$ beta\}-cell apoptosis and islet inflammation. J Biol Chem 2011;286:929-41.

41. Medley SC, Rathnakar BH, Georgescu C, et al. Fibroblastspecific Stat1 deletion enhances the myofibroblast phenotype during tissue repair. Wound Repair Regen 2020;28:448-59.

42. Lun $W, W u X$, Deng Q, et al. MiR-218 regulates epithelial-mesenchymal transition and angiogenesis in colorectal cancer via targeting CTGF. Cancer Cell Int 2018;18:83.

Cite this article as: $\mathrm{Wu} \mathrm{J}$, Qin Y, Li Z, Li J, Li L, Tao S, Liu D. Comprehensive analysis of lncRNA and miRNA expression profiles and ceRNA network construction in negative pressure wound therapy. Ann Transl Med 2021;9(17):1383. doi: 10.21037/ atm-21-3626
43. Han S, Kong YC, Sun B, et al. microRNA-218 Inhibits Oxygen-induced Retinal Neovascularization via Reducing the Expression of Roundabout 1. Chin Med J (Engl) 2016;129:709-15.

44. Wu C, Xue Y, Wang P, et al. IFN- $\gamma$ primes macrophage activation by increasing phosphatase and tensin homolog via downregulation of miR-3473b. J Immunol 2014;193:3036-44.

45. Li P, Sun N, Zeng J, et al. Differential expression of miR$672-5 \mathrm{p}$ and miR-146a-5p in osteoblasts in rats after steroid intervention. Gene 2016;591:69-73.

(English Language Editors: B. Maizey and J. Gray) 


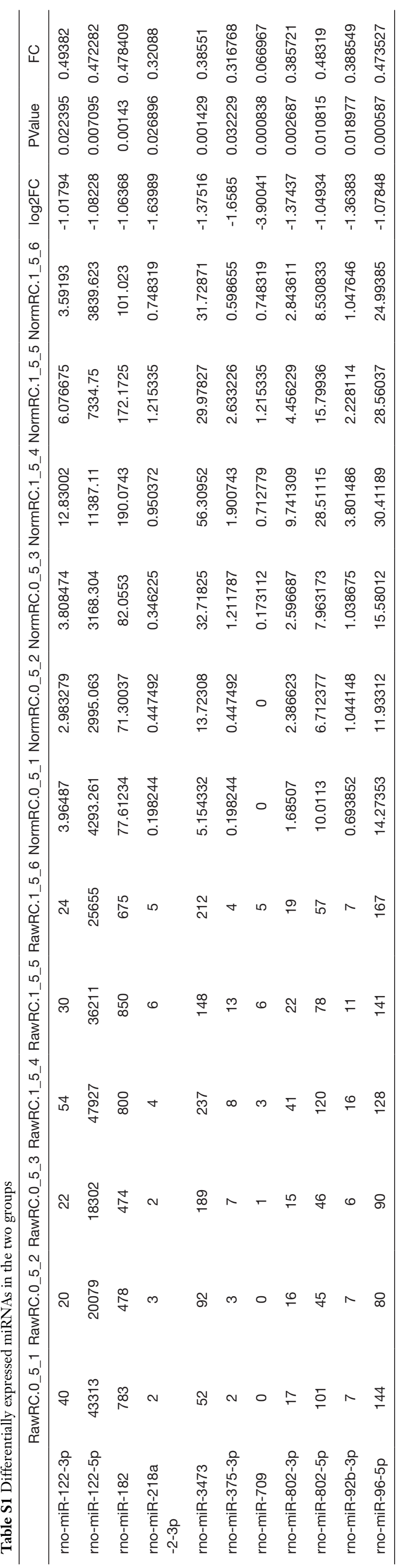


Table S2 The probe sequences and primers for qRT-PCR in the experiment

\begin{tabular}{ll}
\hline Gene & Primer sequence \\
\hline MERGE.27685.3 & F: TTCCTCTGCGCCAGAAGAAG; R: TCTTGCCCAAACACCTGGTT \\
NONRATT007883.2 & F: GTCCCTAAGCCTAACCTGCG; R: GTCTTGTCCGAGGTGTGAGG \\
MERGE.6154.9 & F: CACAGCCACTGCACACTTC; R: CCTCAGGCTGATGGCAAAGA \\
MERGE.18820.19 & F: GTGAAACGTGAGGGGCAAAC; R: CTCTCCCTTCACCTTGCAGG \\
GAPDH & F: TCCAGTATGACTCTACCCACG; R: CACGACATACTCAGCACCAG \\
\hline
\end{tabular}
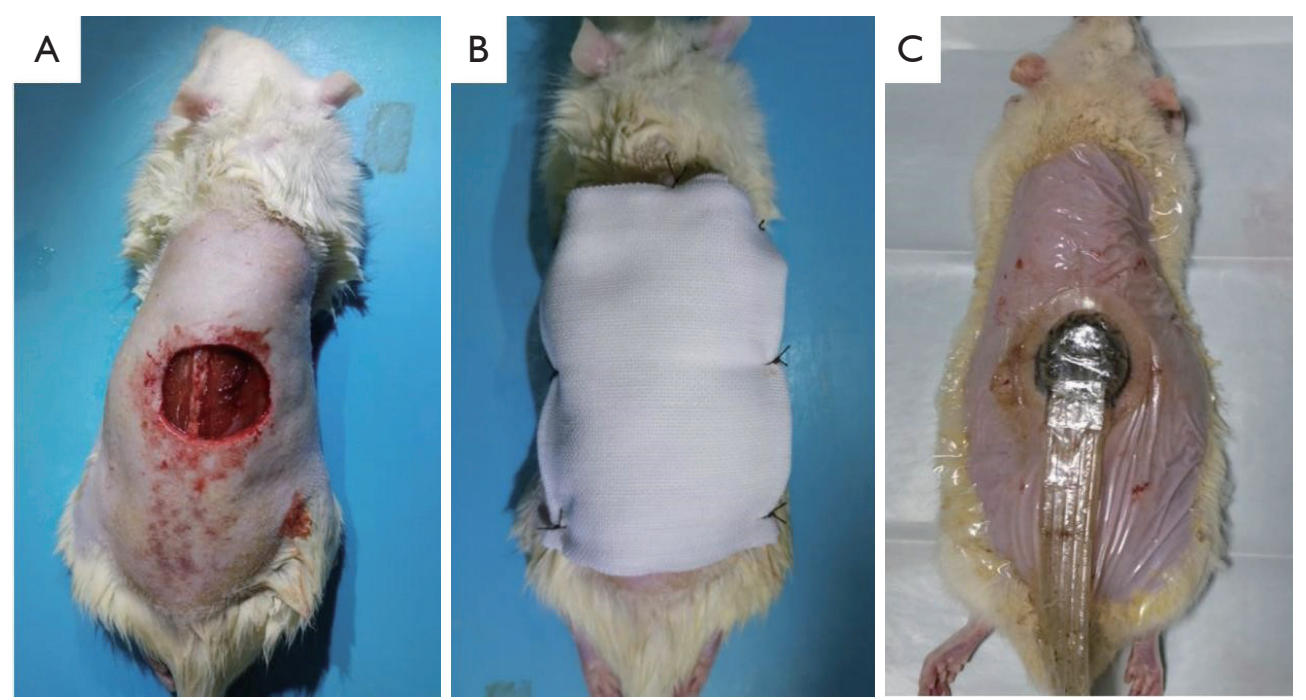

Figure S1 General observation of fresh wound (A), gauze group (B) and NPWT group (C). NPWT, negative pressure wound therapy. 